\title{
Rechtsgeschichte
}

\section{Marcel Senn}

\section{Wissenschaftsgeschichte als Mittlerin zwischen} Öffentlichkeit und Recht 


\section{Wissenschaftsgeschichte als Mittlerin zwischen Öffentlichkeit und Recht*}

I. Rechtswissenschaft zwischen Himmel und Erde

Müssen wir Psychophoros erneut bemühen, um uns die Unvollkommenheit unseres menschlichen Denkens vor Augen zu

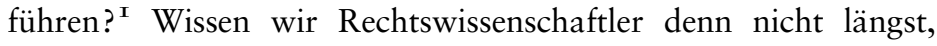
dass wir gerne in einem Begriffshimmel leben? Natürlich wissen wir das. Aber wissen wir nicht auch, dass diese himmlischen Begriffs-Götzen alles andere als reine Lichtgestalten, sondern meist nur kleine Teufel sind? Selbst das wissen wir, dass der Himmel der Wissenschaft nicht so hell und klar ist, wie wir Rechtswissenschaftler dies gerne hätten.

Vergewissern wir uns also durch einen kritischen Blick in diesen Himmel. In einer der vielen himmlischen Hallen zur »Großen Erkenntnis « strahlen so schöne Begriffe wie »Wissenschaftsgeschichte «, »Öffentlichkeit « und »Recht ", die sogleich unsere Aufmerksamkeit fordern. Bei genauerem Hinsehen erweisen sie sich jedoch nicht als jene erhofften Lichtgestalten, sondern als unvollkommenere Kreaturen, wie dies bei alltäglichen Worten, die in aller Munde und Schreibe kursieren, der Fall ist. Aber und das ist das Faszinosum - zahlreiche Wissenschaftler halten sie für Lichtgestalten und glauben deshalb zu wissen, wovon die Rede und Schreibe ist. Einige haben sich ja auch, weiß Gott, redlich bemüht, ihre Worte zu definieren, sie gleichsam dogmatisch einzuzäunen. Doch wie schwierig, ja geradezu aussichtslos solch allzu menschliches Unterfangen letztlich ist, lehren einen die schwergewichtigen Worte selbst, wenn sie in kleinlaute Klangformhülsen gepresst werden sollen und sich schlicht sperren. So lehren sie uns die Einsicht, dass wir eben doch nur auf Erden und nicht im Begriffshimmel leben, wenn wir in wissenschaftlichen Büchern und Artikeln von Worteingrenzungen lesen wie der "Wissenschaftsgeschichte" als der Entwicklung von nachvollziehbarer Wissensproduktion unter besonderen Rahmenbedingungen oder von der »Öffentlichkeit « als dem Gemeinplatz staatlicher Allgemeinheit oder vom »Recht «, das - nach implizit kantianischer

\footnotetext{
* Ich danke meinen Assistierenden lic. iur. Mihai Vladescu und Katharina Niederberger für die Materialbeschaffung und das kritische Feedback.

I Vgl. dazu: R. v. JHERing, Scherz und Ernst in der Jurisprudenz [I. Aufl. I884], hg. von M. LeITNER, Wien 2009, 249.
} 
Denklinie verkürzt - als legitimer äußerer Zwang erscheint. Und manchmal hinkt ein Eingeständnis hintendrein, dass diese verengten Begriffe auch eine umfassendere Bedeutung haben könnten.

Diese soeben festgestellte Eigenart des Wissenschaftsbetriebs mag Wissenschaftsneulinge erschrecken und Wissenschaftsgläubige irritieren. Für die Abgebrühten und Ganzkalten ist solche Faktizität normal. In ehrlicher Diktion wird dies als offener Diskurs über Grundlagenfragen apostrophiert, was etwa soviel besagt, dass es sich um Umschreibungen von Phänomenen handele, mit denen wir trotz aller wissenschaftlicher Ernsthaftigkeit und nächtelangen Bemühungen nicht weiter gelangt sind. Dies einzugestehen, löst freilich Beklemmung aus. Doch ist die Unschärfe der Begriffe unserer Sprache inhärent. Lassen wir also Psychophoros getrost weiter ruhen.

\section{Wozu noch Wissenschaft im gelobten Paradies} der Wissensgesellschaft?

Die aus wissenschaftlichem Anspruch nach Klarheit entstehende Spannung im Verhältnis zur Unschärfe unserer Begriffe löst oder verstärkt sich im Zusammenhang mit dem Verständnis darüber, was die eigentliche Aufgabe der Wissenschaft sei. Sie verstärkt sich, wenn vermeint wird, die Aufgabe der Wissenschaft bestehe vor allem darin, möglichst viel verwendbares, also nutzfertiges Wissen zu produzieren, das in kleine Portionen von Begrifflichkeiten abgepackt sein müsse. Dies ist freilich auch eine Form von Klarheit. Doch führt bloße Wissensproduktion, wie wir wissen, nicht ins Ziel. Die Spannung löst sich indes, wenn das Ziel der Wissenschaft in der intellektuellen Vorgehensweise gesehen und damit von der auf den Nutzen ausgerichteten Funktion der Wissenschaft unterschieden wird.

Das Ziel oder die Hauptaufgabe der Geistes- und Kulturwissenschaft, die hier im Fokus steht, war es seit je, den aktuellen Wissensbestand, die Entstehung von neuem Wissen und die Bedeutung dieses Wissens zu reflektieren. Entsprechend diesem Wissenschaftsverständnis war und ist das unmittelbar verwertbare Wissen nicht das Ziel, sondern eine Funktion, die sich ergibt. Mit Aufkommen des Positivismus und Erstarken des Utilitarismus im Industriezeitalter des I9. Jahrhunderts wurde jedoch auch die Rechtswissenschaft immer ergebnisorientierter auf das vorder- 
gründig Verwendbare ausgerichtet. Insofern hat sich das Verhältnis von Haupt- und Nebenaufgabe der Wissenschaft verkehrt. Seither schafft der wissenschaftliche Positivismus, den die Kontextfragen nach den Bedingungen und Auswirkungen der Wissenschaftsproduktion nicht eigentlich interessieren, konkretes Wissen, das nutzbar ist; er schafft somit die für gewisse Zeit sicheren Wissensbestände einer Disziplin, die ihr Wissen der Gesellschaft zur Verfügung stellt. Der Positivismus stellt damit nicht die Wissenschaft selbst dar, denn dafür fehlt ihm die Kraft der kritischen Selbstreflexion. Er ist indessen eine Voraussetzung für gute, seriöse Wissenschaft, die Fakten erstellt.

Wenn es also in erster Linie um wissenschaftliche Reflexion und erst in zweiter um wissenschaftliche Produktion geht, so gibt es dafür einen sachlichen Grund: Ein Produkt ist so lange tauglich, als seine vormaligen Produktionsbedingungen mit den aktuellen Gegebenheiten wenigstens in den Grundzügen weiterhin übereinstimmen. Wenn wir aber feststellen, dass ein bestimmtes Wissen obsolet wird und der Geschichte anheim fällt, so liegt der Grund dafür eben darin, dass sich dessen Voraussetzungen zwischenzeitlich grundlegend verändert haben. Es entsteht dadurch ein Bruch zwischen den Bedingungen von damals und heute, weil konkretes Wissen stets ein definierter Ausschnitt aus einem Gesamtzusammenhang des Wissensmöglichen ist. Dieser Gesamtzusammenhang korrespondiert mit der Komplexität von Wirklichkeit, und diese lässt sich letztlich immer nur intuitiv als »ein Ganzes « begreifen und folglich nur mit vagen Ausdrücken - wie eingangs geschildert begrifflich erfassen und wiedergeben. Da sich die Elemente im Gesamtzusammenhang aber dauernd verändern und damit auch die Bedingungen für wissenschaftliche Aussagen, wird dieses Wissen brüchig. Wissenschaftliche Reflexion nimmt somit diese »Brechung « vorweg und legt dadurch die - ohnehin meist fragilen Wissensbestände mitsamt ihren Voraussetzungen offen, unter denen sie entstanden sind.

Bereits an dieser Stelle zeichnet sich ab, welche Bedeutung der Wissenschaftsgeschichte zukommt. Aufgrund des vorerwähnten Gedankenganges wird klar, weshalb sich die Wissenschaft in erster Linie mit »dem Ganzen" auseinandersetzen muss und erst an zweiter Stelle die Auseinandersetzung mit einzelnen Beständen von Wissen zu betreiben hat. ${ }^{2}$ Wissenschaft ist und bleibt stets ein Reflexionsversuch in Aussagen über Wirklichkeit. Die Behaup-

2 Vgl. dazu: G. LINDE, Wissen-

schaftstheorie, in: Religion und Geschichte in der Gegenwart, hg. von H.D. BETZ, D. S. BRowning, B. JANOWSKI, E. JÜNGEL, Bd. 8, 4. A. Tübingen 2008, I66I ff. 
tung, Wissenschaft könne ein Phänomen abschließend (statt annähernd) erklären, ist, weil sie dies nie kann, nur ein Ausdruck eines wohl übermütigen Verständnisses einzelner Wissenschaftler; dieses Verständnis unterscheidet sich von einer engen Sicht auf die Welt indes kaum. Daher gibt die Wissenschaft einer Sache erst ibren Sinn, weil die einzelnen Wissenselemente erst aus ihrem Gesamtzusammenhang heraus begreifbar sind. Die Wissenschaft im Sinne seriöser Reflexion erklärt ihre jeweilige Sinngabe daher immer als eine von vielen plausiblen Möglichkeiten. Dadurch wird eine Offenheit im wissenschaftlichen Erklärungsvorgang selbst erzeugt und gewährleistet, um anderen (sogar noch nicht vorgetragenen) Erklärungen bewusst Raum zur Artikulation zu belassen. ${ }^{3}$ Insofern unterscheidet sich diese wissenschaftliche Sinngebung durch ihren kritischen Intellektualismus von Doktrin, Allerweltserklärung oder Religion. Wissenschaft ist erst dann Wissenschaft, wenn sie sich durch ihre Art und Weise der Sinngabe, mithin als Akt des hinterfragenden philosophischen Intellekts, gegenüber Doktrin und Dogma klar abgrenzt. Der größte Irrtum in der Wissenschaft, den sich diese allerdings immer wieder leistet, besteht gerade darin, selber zu glauben (!) und anderen weiszumachen, sie erkläre die Welt oder Wirklichkeit im Sinne irgendeiner Gesetzesmäßigkeit oder irgendeines Systemverstehens abschließend.

Gerade unsere viel gerühmte Wissensgesellschaft ${ }^{4}$ von heute bleibt im vorerwähnten Sinn ein unvollkommenes Gehäuse, in dem nicht der Intellekt, sondern das Vielwissen zählt. Diese »Wisserei « ist aber nur eine neue Form von Armut im Geiste. Sie entspricht jenem Enzyklopädismus, der stets auf der Stufe der Voraussetzung zur kritischen Wissenschaft verharrte. In dem Sinn ist die Wissensgesellschaft ein Ausdruck für eine stehen gebliebene und letztlich anti-intellektuelle Form von Gesellschaft. Dass Wissenschaft noch Grundlegenderes darüber hinaus forderte, nämlich eine bestimmte reflexive Haltung, ist hier allerdings nicht zu erörtern. ${ }^{5}$

\section{Einsichten aus Wissenschaftsgeschichte}

In der Wissenschaftsphilosophie und Wissenschaftstheorie übernimmt die Disziplin der Wissenschaftsgeschichte, wie zuvor angemerkt, die Aufgabe der Analyse der Wechselbeziehungen von konkreter Wissensproduktion und begrifflicher Wissensverarbeitung. Die Wissenschaftsgeschichte bringt somit ein klärendes Licht
3 Vgl. dazu: H. J. SANDKÜHLER, Kritik der Repräsentation. Einführung in die Theorie der Überzeugungen, der Wissenschaftskulturen und des Wissens, Frankfurt am Main 2009, insb. 2I-25.

4 Vgl. dazu: U. H. BitTlingmaYeR, H. TunCER, Die Wissensgesellschaft - eine folgenschwere Fehldiagnose, in: Handbuch Wissensgesellschaft. Theorien, Themen und Probleme, hg. v. A. EngeL-
HARDT und L. KAJETZKE, Bielefeld 2OIO, insb. 352 ff.; ST. BÖSCHEN, Wissenschaft und Gesellschaft, in: Handbuch Wissenssoziologie und Wissensforschung, hg. von R. SCHÜTZEICHEL, Konstanz 2007, insb. 756 ff.: Danach läuft die gesellschaftliche Pluralisierungsstrategie nicht nur Gefahr in Relativismus zu zerfallen, sondern gleichzeitig auch durch ein

Ökonomisierungs- bzw. Mediali- sierungsregime aufgefangen zu werden.

5 Vgl. dazu: M. SenN, Rechtswissenschaft ohne reflexive Haltung?, in: Kommunikation. Festschrift für Rolf H. Weber zum 60. Geburtstag, hg. von R. SETHE, A. Heinemann, R. M. Hilty, P. Nobel, R. Z̈̈сH, Bern 2OI I, 913-929. 
6 Die Dialektik zwischen den universitären Produktionsbedingungen und den gesellschaftlichen Mentalitätseinflüssen als grundlegenden Einflussfaktoren auf die Wissenschaftsproduktion wäre ein lohnendes, wenn auch anspruchsvolles Untersuchungsvorhaben. Zum ersten Aspekt vgl.: Geschichte der Universität in Europa, hg. von W. RÜEGG, Bd. III. Vom I9. Jahrhundert zum Zweiten Weltkrieg (I800-I945), München 2004, insb. 97-I26, zum zweiten Aspekt vgl. P. DinzeLBACHer, $\mathrm{Zu}$ Theorie und Praxis der Mentalitätsgeschichte, in: Europäische Mentalitätsgeschichte, hg. von P. Dinzelbacher, 2. Aufl. Stuttgart 2008, XVII-XLIII.

7 Vgl. dazu: K.-F. Wessel, Thomas DIESNER, Wissenschaftstheorie und Wissenschaftsgeschichte, in: Wissenschaftstheorie, hg. von D. Horster, W. Jantzen, Stuttgart 20IO, $55 \mathrm{f}$.

8 Vgl. dazu: P. Wehling, Im Schatten des Wissens? Perspektiven der Soziologie des Nichtwissens, Konstanz 2006, insb. I67 ff.

กั

9 Vgl. dazu das polemisch-köstliche Essay von N. Postman: Wir amü-

in das Verhältnis von Haupt- und Nebenaufgabe der Wissenschaft, indem sie den Bereich von produktivem Positivismus und kritischer Hinterfragung des Wissens ausleuchtet und deutlich macht, wie die Zusammenhänge des aktuellen Wissens (zu einem bestimmten Gegenstande) konstituiert sind. Indem sie die Entstehungsprozesse eines bestimmten Wissens erklärt, vermittelt sie zwischen dem nutzbaren Wissen und seinen Produktionsbedingungen, ${ }^{6}$ klärt dadurch auf, wie und weshalb Wissen in der Art, wie es vorliegt, entstanden und in welchem Kontext dieses Wissen (nur) zu verstehen ist. ${ }^{7}$ Damit öffnet sich ein Fenster auf eine Vielfalt von Sichtweisen, weshalb es insbesondere auch einer integrierten Perspektive des Nichtwissens bedarf. ${ }^{8}$ In dem Sinne bildet die Wissenschaftsgeschichte die Grundlage der Wissenschaftsphilosophie, die auch untersucht, wie viel Nichtwissen faktisch den Hintergrund einer bestimmten Wissens- und Wissenschaftskultur - gleichviel, ob gewollt oder nicht gekonnt - bildet.

Solche Aufklärungsarbeit wurde in den I960er bis 8oer Jahren öfters gepflegt, doch mit Zunahme der Wissensgesellschaft immer mehr verdrängt. Dies hat verschiedene Gründe; ich nenne die drei meines Erachtens wichtigen:

I. In weiten Kreisen besteht ein eher sorgloses Verständnis betreffend den Zusammenhang von Wissen und Welt. Mit Aufkommen der Vorstellung einer Wissensgesellschaft erhält der Enzyklopädismus oder das Vielwissen, wie erwähnt, eine positive Bewertung. Diese Wissensgesellschaft gründet auf einem Weltverständnis, wonach alles mach-, konsumier- und reproduzierbar ist, heute umso mehr, weil die Informationen über die Welt aus dem Internet jederzeit verfügbar sind. Und zugleich soll solches Wissen obendrein unterhaltend und vor allem billig sein. ${ }^{9}$

2. Dahinter steht ein kurzfristiges Nützlichkeitsdenken, dem die Strategie heutiger Wissenschaftspolitik meist entspringt. Im universitären Bereich wurden Forschungs- und Lehrstellen im Grundlagenbereich der Rechtswissenschaften ${ }^{10}$ während der letzten zwanzig bis dreißig Jahre massiv gekürzt oder umgewidmet. Im schweizerischen Lehrbetrieb - um ein Beispiel zu nennen erfolgte die Zielverlagerung vom Lernziel des selbständigen Erarbeitens und des autonomen Denkens in den »Punkteerwerbslehrbetrieb " nach Maßgabe des sogenannten Geistes von Neu-

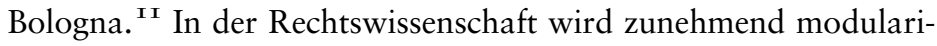
siertes Wissen abgeprüft. Die Kontextualisierung und Vernetzung

sieren uns zu Tode. Urteilsbildung im Zeitalter der Unterhaltungsindustrie [1985], Frankfurt am Main, I7. Aufl. 2006.

Io $\mathrm{Zu}$ einigen Gründen siehe: M. SENN, Rechtswissenschaft und Geschichte, in: Interdisziplinarität in den Rechtswissenschaften Innen- und Außenperspektiven, hg. von K. Stephan, M. AnderHALDEN. Der Band erscheint bei Mohr \& Siebeck, Tübingen 20I I.
Siehe dort unter Ziff. 5 bei Anm. 22.

I I Vgl. M. SenN, Rechtswissenschaft nach der Bologna-Reform, in: Rechtswissenschaft. Zeitschrift für rechtswissenschaftliche Forschung I (20IO) Heft 2, 2I 8-224. Der Beitrag ist aus der Perspektive als Dekan der Rechtswissenschaftlichen Fakultät der Universität Zürich verfasst. 
der einzelnen Modulinhalte beziehungsweise das reflexive Denken über die Lerninhalte werden immer mehr marginalisiert. Dadurch bewältigen Anpassungswillige, manchmal auch Auswendiglerner, das Studium öfters mit größerem Erfolg als jene Studierenden, die das Recht verstehen und hinterfragen wollen. Was dies für die Gesellschaft letztlich bedeutet, lässt sich nur erwägen bzw. erahnen.

3. Damit einher geht eine weitere Beobachtung: Die Fähigkeit zur kritischen Aufarbeitung von intellektuellem und kontextualisiertem Wissen geht zunehmend verloren, und die seriöse und mühevolle Recherche wird in den Medien wie in der Wissenschaft immer weniger verlangt. Denn dies ist stets auch eine Ressourcenfrage. »Ökonomisierendes « Denken bestimmt immer stärker unsere gesamte Wissenskultur.

In dem Sinne kann die Wissenschaftsgeschichte gerade als Dorn im Fleische der » produktiven Wissenschaft « wirken, und dies umso mehr, je stärker sie aus der Tradition der Wissenschaftsphilosophie die Produktionsbedingungen von Wissen und die Wissenskultur hinterfragt.

\section{Verantwortlichkeit der Öffentlichkeit}

Wenn die wissenschaftliche Selbstreflexion, unterstützt durch die Wissenschaftsgeschichte, in der soeben beschriebenen Offenheit gelingt, dann ist die Wissenschaft dadurch auch Gegenstand der Öffentlichkeit. Denn im Sinne der Selbstreflexion beeinflusst und fördert die Wissenschaft ebenfalls die Entwicklung der Zivilgesellschaft. Dadurch wird die auf tatsächlichem oder auch nur vorgegebenem Wissen aufgebaute gesellschaftliche Macht zwar nicht durchbrochen, jedoch durchsichtiger gemacht. ${ }^{12}$ Diese Schnittstelle von Wissenschaft und Gesellschaft zeigt, dass es zwar zuerst immer um die Qualität der Wissenschaft selbst geht, damit aber stets auch die Frage nach der Qualität der Gesellschaft gestellt ist, für die sie das konkrete und nützliche Wissen erzeugt.

Diese Öffnung der Wissenschaft auf die gesellschaftliche Öffentlichkeit hin sollte allerdings nicht dahingehend missverstanden werden, dass sich die Öffentlichkeit nun in irgendeiner Weise in die Wissenschaft einzumischen hätte, fehlt es ihr doch am Expertenwissen, um das es in den wissenschaftlichen Prozessen geht. Dass jedoch genau diese Situation aktuell zum Trend wird, soll nicht verkannt werden: Die Wissenschaft von heute wird zunehmend

I2 Vgl. dazu: F. Hanschmann,

Der Begriff der Homogenität in

der Verfassungslehre und Europa-

rechtswissenschaft, Berlin u. a.

2008, 196-207 und 212. 
politisch bestimmt, wie sich dies die vorerwähnte Umstellung des Lehrbetriebs - etwa in der Rechtswissenschaft - nach betriebswirtschaftlichen Bedürfnissen und europäischer Gleichmacherei im Geiste der Bologneser Kompatibilität zeigt.

Das Postulat der Öffnung der Wissenschaft kann daher nur meinen, dass sich die Wissenschaft der Öffentlichkeit gegenüber in der Weise öffne, ${ }^{\mathbf{1}}{ }^{3}$ dass sie ihr nicht nur das konkret verwertbare Wissen verschafft, sondern diese Gesellschaft als die Öffentlichkeit zugleich auch daran erinnert, dass sie selbst mit diesem Wissen kritisch und sorgsam umzugehen hat. Denn die Gesellschaft, nicht die Wissenschaft entscheidet letztlich darüber, was mit dem Wissen $\mathrm{zu}$ geschehen hat, auch wenn in der gegenwärtig verkehrten Welt die Verantwortlichkeit häufig an die Wissenschaft delegiert wird.

\section{Das Recht und seine Geschichte}

Seit dem I8. Jahrhundert bildete sich in der Rechtswissenschaft die Tendenz zur positivistischen Perspektive heraus, die in der zweiten Hälfte des I9. Jahrhunderts noch akzentuiert wurde. ${ }^{\text {I4 }}$ Die positivistische Perspektive revolutionierte eine mehr als zweitausendjährige Tradition eines letztlich (metaphysisch) durch die Ethik bestimmten Rechtsdenkens durch einen - äußeren methodologischen Prinzipien folgenden - Rechtsformalismus. Eine Erweiterung dieser positivistischen Perspektive erfolgte sodann durch Formulierung von Menschenrechtserklärungen und Grundrechtskatalogen während des I9. und 20. Jahrhunderts. Dadurch wurde der allzu enge Rechtsbegriff ein Stück weit wieder korrigiert. Doch gerade die Korrektur des Positivismus durch ein Nachschieben einer in der juristischen Semantik des Positivismus gehaltenen Verrechtlichung »fundierender « Ethik lässt ja nur evident werden, dass und wie sehr die gepriesene Trennung von Recht und Ethik einen überzogenen Rigorismus darstellt.

Diese Trennung war - historisch besehen - das Ergebnis eines durchaus nachvollziehbaren Vorganges. Man suchte Rechtssicherheit, die nach den konfessionalisierten Kriegen und im Verhältnis des Individuums zum Obrigkeitsstaat etabliert werden sollte. Der Rechtsformalismus sollte diese Anliegen realisieren helfen, und das Recht selbst dadurch gerechter werden. Formales Recht sollte die streitigen Punkte einer konfessionalisierten Ethik und Religion mit Bezug auf das Recht jederzeit abweisen und den Bürgern sollte

I3 In diesem Sinne auch H. J. KLEINSTEUBER, Öffentlichkeit, in: Kleines Lexikon der Politik, hg. von D. Nohlen, F. Grotz, 4. Aufl. München 2007, 373.

I4 Vgl. J. SchröDER, Recht als Wissenschaft. Geschichte der juristischen Methode vom Humanismus bis zur historischen Schule (I $500-$ I850), München 200I. 
gleichzeitig mehr Freiheit gewährt werden. Indem das Recht im Namen der neuen Freiheit streng auf die Vorstellung eines legitimen Zwanges reduziert wurde, glaubte man dies bewerkstelligen zu können. Diese Vorstellung war jedoch noch der Gedankenwelt des Obrigkeitsstaats verhaftet, dem - so schien es - nur durch solch eine äußere Objektivität eines » legitimen Zwangsrechtes « begegnet werden konnte. In der Folge wurde die formale Rechtsauffassung als das allgemeingültige Recht inthronisiert und im Europa der ersten Hälfte des 20. Jahrhunderts, insbesondere in Deutschland und der UdSSR, durch anti-ethische und inhumane Systeme der Staatsdominanz zu einem trivialen Organisationsmechanismus der Staatstechnokratie herabgewürdigt. ${ }^{15}$ Mit Ende des Zweiten Weltkrieges war zwar eine politische Zäsur erreicht, der aber, wie wir wissen, keine wirksame wissenschaftliche Erneuerung folgte. ${ }^{{ }^{\mathbf{6}}}$ Es kann daher wenig erstaunen, dass sich gewisse Trends des vormaligen Macht- und Dominanzdiskurses, des Formalismus und des Kollektivismus auf der Ebene der modernen Rechtstheorien ${ }^{17}$ gleichsam auf abstrakter Ebene - zum Teil fortsetzte. Am deutlichsten lässt sich diese schleichende Übertragung dort wahrnehmen, wo der selbständig denkende Mensch bloß als störender Fremdkörper gegen ein propagiertes System aufgefasst wird. Den formalmethodologischen Trends, welche die Macht, den Formalismus und das System repräsentier $(\mathrm{t})$ en, muss daher entschieden entgegengehalten werden, dass das Recht - in der antik-europäischen Tradition seit Aristoteles - auf die Selbstorganisation der Menschen unter sich ausgerichtet war und ist, und dies alleine derentwillen, nicht eines Systems oder Staates wegen. Unter diesem Gesichtspunkt lässt es sich heute noch rechtfertigen zu sagen, das Recht habe der Ethik zu folgen. ${ }^{\text {I }}$

6. Wissenschaftskritische Reflexion des Rechts auf historischer Grundlage

Unter diesem Aspekt kommt der Wissenschaftsgeschichte somit eine entscheidende aufklärende Bedeutung für das Recht, seine Wissenschaft und die Gesellschaft zu. ${ }^{\text {I9 }}$ Michael Stolleis hat mit seiner "Geschichte des öffentlichen Rechts in Deutschland « nicht nur gezeigt, wie dieser innerwissenschaftliche Diskurs aufzuarbeiten ist, sondern er hat auch einen Weg gewiesen, wie das Verhältnis von Wissenschaftsgeschichte, Recht und Öffentlichkeit für die

I 5 Vgl. dazu: M. SENN, Rechtsgeschichte - ein kulturhistorischer Grundriss, 4. Aufl. Zürich, Basel, Genf 2007, 40I ff.

I 6 Vgl. dazu: B. RüTHERs, Geschönte Geschichten - geschonte Biographien. Sozialisationskohorten in Wendeliteraturen: Ein Essay, Tübingen 200I, insb. 72-97.

I7 Vgl. dazu: H. A. Hesse, Recht, in: Lexikon Soziologie und So- zialtheorie, hg. v. S. FARZIN u. A., Stuttgart 2008, $235 \mathrm{ff}$.

I 8 Vgl. dazu: R. Zippelius, der in seiner ebenso profunden wie konzisen und weisen »Geschichte der Staatsideen « ( Io. A. München 2003) bemerkt (S. 35), dass auch der Positivist zugeben müsse, dass jede Gemeinschaft unabhängig von Gesetzen gewisse Verhaltensregeln als gerecht bzw. ungerecht ansehe. Dieses Argument hat auch schon R. Dworkin in Abgrenzung zum Positivismus eines Hart vertreten (vgl. Taking Rights Seriously, Cambridge 1977 , insb. $47 \mathrm{ff}$.).

I9 Vgl. dazu den ausgezeichneten Artikel von W. KROHN, Wissenschaftsgeschichte, in: Enzyklopädie Philosophie, hg. von H. J. SANDKÜHLER, Hamburg 20IO, Bd. 3, 3030 ff. 
gesellschaftliche Entwicklung fruchtbar genutzt werden kann. Indem der Rechtshistoriker, so schrieb er I988 einleitend, die geschichtliche Bedingtheit der Probleme darlege, löse er sie zwar nicht, ja womöglich zeige er sogar bloß deren Unlösbarkeit auf, doch durch die Erschließung ihrer historischen Dimension würden die Fragen betreffend das Recht begreifbarer gemacht und die Gründe für bislang gescheiterte Lösungsvorschläge verständlicher. Dadurch würde zumindest die aktuelle Gesetzgebung von einer verfehlten Lage- und Problembeurteilung entlastet. ${ }^{20}$

Im Lichte dieser Ausführungen sollte folglich der Sinn der Juristenausbildung unter folgenden zwei Aspekten erneut diskutiert werden: I. Welche Ziele sollen mit der juristischen Ausbildung erreicht werden? 2. Ist die Universität eine Schule, welche die Praxis des gerade gängigen Rechtsdiskurses zu vermitteln hat, oder ist sie ein autonomes Forum, welches aufklärend wirken und auf die grundlegenden Fragen nach der Gerechtigkeit des Rechts in der Gesellschaft und auch nach den Gründen des Scheiterns von ethischen Lösungsvorschlägen eingehen soll?

Solange die Universität keine kritische, sondern im Sinne von Neu-Bologna eine mehr technokratische Funktion wahrzunehmen hat, bleibt sie fast zwangsläufig bloße Stoffvermittlerin dogmatischen Wissens. Sofern sie indessen diese aufklärende Funktion übernehmen und erfüllen soll, muss den Grundlagenfächern, vorab der Geschichte des Rechts, der Geschichte seiner Wissenschaft sowie insbesondere auch der kritischen Philosophie - mithin dem reflexiv-historischen Rechtsdenken -, vermehrte Präsenz in der Juristenausbildung unbedingt wieder eingeräumt werden.

Marcel Senn

20 M. Stolleis, Geschichte des öffentlichen Rechts in Deutschland, Bd. I, München I988, 56 . 